\title{
Loud Noises Lead To Dementia
}

\section{Robert Skopec,}

Free-Lance Science Writer, Dubnik, Slovakia.

Corresponding Author: Robert Skopec, Free-Lance Science Writer, Dubnik, Slovakia.

Received date: October 23, 2021; Accepted date: December 21, 2021; Published date: January 03, 2022

Citation: Robert Skopec, (2022). Loud Noises Lead To Dementia. J. Neuroscience and Neurological Surgery. 11(2); DOI:10.31579/2578$8868 / 208$

Copyrights: (C) 2022 Robert Skopec, This is an open-access article distributed under the terms of The Creative Commons Attribution License, which permits unrestricted use, distribution, and reproduction in any medium, provided the original author and source are credited.

\begin{abstract}
Dementia is an umbrella term for a collection of symptoms that are caused by disorders affecting the brain and impact on memory, thinking, behaviour and emotion. The most common is Alzheimer's disease, which affects 50$60 \%$ of people with dementia. Other types of dementia include vascular dementia, Lewy body dementia and frontotemporal dementia. Dementia can also sometimes affect people who are under the age of 65 . This is known as young onset dementia. Our brains are made up of over 86 billion nerve cells - more than the stars in the Milky Way. Dementia damages nerve cells so they are no longer able to communicate effectively and this impacts on how our body functions.
\end{abstract}

Key words: alzheimer's disease; pulmonary disease

\section{Introduction}

The specific symptoms a person living with dementia experiences will depend upon what parts of the brain are affected and/or the specific disease that is causing their dementia. Symptoms may include:

- $\quad$ loss of memory

- difficulty in finding the right words or understanding what people are saying

- difficulty in performing previously routine tasks

- $\quad$ personality and mood changes

Although each person will experience dementia in their own way, eventually those affected will be unable to care for themselves and need help with all aspects of daily life. Dementia is the leading cause of disability and dependency among the elderly.

Dementia affects more than $\mathbf{5 0}$ million people worldwide, with a new case of dementia occurring somewhere in the world every 3 seconds. Dementia can also affect individuals under the age of 65 (young onset dementia). Greater awareness and understanding of dementia is important to challenge the myths and misconceptions that surround the condition.

There is currently no cure for most types of dementia, but treatment and support are available.

Key facts
- Dementia is a syndrome in which there is deterioration in cognitive function beyond what might be expected from the usual consequences of biological ageing.

- Although dementia mainly affects older people, it is not an inevitable consequence of ageing.

- Currently more than 55 million people live with dementia worldwide, and there are nearly 10 million new cases every year.

- Dementia results from a variety of diseases and injuries that primarily or secondarily affect the brain. Alzheimer's disease is the most common form of dementia and may contribute to 60$70 \%$ of cases.

- Dementia is currently the seventh leading cause of death among all diseases and one of the major causes of disability and dependency among older people globally.

- Dementia has physical, psychological, social and economic impacts, not only for people living with dementia, but also for their carers, families and society at large.

Dementia is a syndrome - usually of a chronic or progressive nature - that leads to deterioration in cognitive function (i.e. the ability to process thought) beyond what might be expected from the usual consequences of biological ageing. It affects memory, thinking, orientation, comprehension, calculation, learning capacity, language, and judgement. Consciousness is not affected. The impairment in cognitive function is 
commonly accompanied, and occasionally preceded, by changes in mood, emotional control, behaviour, or motivation.

Dementia results from a variety of diseases and injuries that primarily or secondarily affect the brain, such as Alzheimer's disease or stroke.

Dementia is currently the seventh leading cause of death among all diseases and one of the major causes of disability and dependency among older people worldwide. Dementia has physical, psychological, social and economic impacts, not only for people living with dementia, but also for their carers, families and society at large. There is often a lack of awareness and understanding of dementia, resulting in stigmatization and barriers to diagnosis and care.

\section{Signs and symptoms}

Dementia affects each person in a different way, depending upon the underlying causes, other health conditions and the person's cognitive functioning before becoming ill. The signs and symptoms linked to dementia can be understood in three stages.

Early stage: the early stage of dementia is often overlooked because the onset is gradual. Common symptoms may include:

- forgetfulness

- $\quad$ losing track of the time

- becoming lost in familiar places.

Middle stage: as dementia progresses to the middle stage, the signs and symptoms become clearer and may include:

- becoming forgetful of recent events and people's names

- becoming confused while at home

- having increasing difficulty with communication

- $\quad$ needing help with personal care

- experiencing behaviour changes, including wandering and repeated questioning

Late stage: the late stage of dementia is one of near total dependence and inactivity. Memory disturbances are serious and the physical signs and symptoms become more obvious and may include:

- becoming unaware of the time and place

- having difficulty recognizing relatives and friends

- having an increasing need for assisted self-care

- $\quad$ having difficulty walking

- $\quad$ experiencing behaviour changes that may escalate and include aggression.

\section{Common forms of dementia}

There are many different forms of dementia. Alzheimer's disease is the most common form and may contribute to $60-70 \%$ of cases. Other major forms include vascular dementia, dementia with Lewy bodies (abnormal aggregates of protein that develop inside nerve cells), and a group of diseases that contribute to frontotemporal dementia (degeneration of the frontal lobe of the brain). Dementia may also develop after a stroke or in the context of certain infections such as HIV, harmful use of alcohol, repetitive physical injuries to the brain (known as chronic traumatic encephalopathy) or nutritional deficiencies. The boundaries between different forms of dementia are indistinct and mixed forms often co-exist.

\section{Rates of dementia}

Worldwide, around 55 million people have dementia, with over $60 \%$ living in low- and middle-income countries. As the proportion of older people in the population is increasing in nearly every country, this number is expected to rise to 78 million in 2030 and 139 million in 2050.

\section{Treatment and care}

There is currently no treatment available to cure dementia. Antidementia medicines and disease-modifying therapies developed to date have limited efficacy and are primarily labeled for Alzheimer's disease, though numerous new treatments are being investigated in various stages of clinical trials.

Additionally, much can be offered to support and improve the lives of people with dementia and their carers and families. The principal goals for dementia care are:

- early diagnosis in order to promote early and optimal management

- $\quad$ optimizing physical health, cognition, activity and well-being

- $\quad$ identifying and treating accompanying physical illness

- understanding and managing behaviour changes

- $\quad$ providing information and long-term support to carers.

\section{Risk factors and prevention}

Although age is the strongest known risk factor for dementia, it is not an inevitable consequence of biological ageing. Further, dementia does not exclusively affect older people - young onset dementia (defined as the onset of symptoms before the age of 65 years) accounts for up to $9 \%$ of cases. Studies show that people can reduce their risk of cognitive decline and dementia by being physically active, not smoking, avoiding harmful use of alcohol, controlling their weight, eating a healthy diet, and maintaining healthy blood pressure, cholesterol and blood sugar levels. Additional risk factors include depression, social isolation, low educational attainment, cognitive inactivity and air pollution.

\section{Social and economic impact}

Dementia has significant social and economic implications in terms of direct medical and social care costs, and the costs of informal care. In 2019 , the estimated total global societal cost of dementia was US\$ 1.3 trillion, and these costs are expected to surpass US $\$ 2.8$ trillion by 2030 as both the number of people living with dementia and care costs increase.

\section{Impact on families and carers}

In 2019, informal carers (i.e. most commonly family members and friends) spent on average 5 hours per day providing care for people living with dementia. This can be overwhelming . Physical, emotional and financial pressures can cause great stress to families and carers, and support is required from the health, social, financial and legal systems. Fifty percent of the global cost of dementia is attributed to informal care.

Fronto-temporal dementias are a rare cause of dementia. They are sometimes

Fronto-temporal dementias are a relatively rare cause of dementia which typically develop at an earlier age than Alzheimer's disease. The frontal lobe of the brain is particularly affected in early stages.

Frontal lobe dementia is caused in a similar way to Alzheimer's disease in that it involves a progressive decline in a person's mental abilities over a number of years. Damage to brain cells is more localised than in Alzheimer's disease and usually begins in the frontal lobe of the brain. 


\section{Symptoms}

- The frontal lobe governs people's mood and behaviour. The person's mood and behaviour may become fixed and difficult to change, making them appear selfish and unfeeling

- The person does not usually have sudden lapses of memory which are characteristic of Alzheimer's disease.

\section{Household air pollution and health}

\section{Key facts}

- $\quad$ Around 3 billion people cook using polluting open fires or simple stoves fuelled by kerosene, biomass (wood, animal dung and crop waste) and coal.

- $\quad$ Each year, close to 4 million people die prematurely from illness attributable to household air pollution from inefficient cooking practices using polluting stoves paired with solid fuels and kerosene.

- Household air pollution causes noncommunicable diseases including stroke, ischaemic heart disease, chronic obstructive pulmonary disease (COPD) and lung cancer.

- Close to half of deaths due to pneumonia among children under 5 years of age are caused by particulate matter (soot) inhaled from household air pollution.

\section{Indoor air pollution and household energy: the forgotten 3 billion}

Around 3 billion people still cook using solid fuels (such as wood, crop wastes, charcoal, coal and dung) and kerosene in open fires and inefficient stoves. Most of these people are poor, and live in low- and middle-income countries.

These cooking practices are inefficient, and use fuels and technologies that produce high levels of household air pollution with a range of healthdamaging pollutants, including small soot particles that penetrate deep into the lungs. In poorly ventilated dwellings, indoor smoke can be 100 times higher than acceptable levels for fine particles. Exposure is particularly high among women and young children, who spend the most time near the domestic hearth.

\section{Impacts on health}

3.8 million people a year die prematurely from illness attributable to the household air pollution caused by the inefficient use of solid fuels and kerosene for cooking. Among these 3.8 million deaths:

- $27 \%$ are due to pneumonia

- $18 \%$ from stroke

- $27 \%$ from ischaemic heart disease

- $20 \%$ from chronic obstructive pulmonary disease (COPD)

- $8 \%$ from lung cancer.

\section{Pneumonia}

Exposure to household air pollution almost doubles the risk for childhood pneumonia and is responsible for $45 \%$ of all pneumonia deaths in children less than 5 years old. Household air pollution is also risk for acute lower respiratory infections (pneumonia) in adults, and contributes to $28 \%$ of all adult deaths to pneumonia.

\section{Chronic obstructive pulmonary disease}

One in four or $25 \%$ of deaths from chronic obstructive pulmonary disease (COPD) in adults in low- and middle-income countries are due to exposure to household air pollution. Women exposed to high levels of indoor smoke are more than twice as likely to suffer from COPD than women who use cleaner fuels and technologies. Among men (who already have a heightened risk of COPD due to their higher rates of smoking), exposure to household air pollution nearly doubles that risk.

\section{Stroke}

$12 \%$ of all deaths due to stroke can be attributed to the daily exposure to household air pollution arising from cooking with solid fuels and kerosene.

\section{Ischaemic heart disease}

Approximately $11 \%$ of all deaths due to ischaemic heart disease, accounting for over a million premature deaths annually, can be attributed to exposure to household air pollution.

\section{Lung cancer}

Approximately $17 \%$ of lung cancer deaths in adults are attributable to exposure to carcinogens from household air pollution caused by cooking with kerosene or solid fuels like wood, charcoal or coal. The risk for women is higher, due to their role in food preparation.

\section{Other health impacts and risks}

More generally, small particulate matter and other pollutants in indoor smoke inflame the airways and lungs, impairing immune response and reducing the oxygen-carrying capacity of the blood.

There is also evidence of links between household air pollution and low birth weight, tuberculosis, cataract, nasopharyngeal and laryngeal cancers.

\section{Disproportionate impact on women}

Globally, dementia has a disproportionate impact on women. Sixty-five percent of total deaths due to dementia are women, and disability-adjusted life years (DALYs) due to dementia are roughly $60 \%$ higher in women than in men. Additionally, women provide the majority of informal care for people living with dementia, accounting for $70 \%$ of carer hours.

\section{Why Women's Brains Are More Vulnerable to Disease, From Anxiety to Alzheimer's}

Changing hormones and chronic stress wreak havoc on the body-but you can fight back.

The alarm bells started going off for neuroscientist Roberta Diaz Brinton, Ph.D., three decades ago, when she saw just how hard women in particular were being hit by Alzheimer's disease. Consider these current stats: Nearly two-thirds of patients diagnosed with the brain disorder are women - a staggering one in five of us will be diagnosed by the time we're 65 - and by 2050 , as many as 9 million women may end up with the disease. It's even worse for African Americans, who are two to three times as likely as non-Hispanic whites to develop Alzheimer's.

But when Brinton, the director of the Center for Innovation in Brain Science at the University of Arizona (and whose work has been supported for years by the Women's Alzheimer's Movement), went looking for answers, she found the status quo response to these stats unsatisfying at best and maddening at worst.

"I kept hearing over and over that it was because women live longer than men," she says. "But we only live about four and a half years longer. That does not explain our twofold greater lifetime risk."

\section{Cold and Flu Remedies}

It also doesn't explain why a number of other brain-health issues affect women far more than men: Women are twice as likely as men to develop certain types of brain tumors, nearly twice as likely to deal with depression, and three times as likely to get headaches. We're also much 
more likely to have a stroke and to develop an autoimmune disorder that affects the brain like multiple sclerosis.

Join the conversation about brain health: Top experts shared their insights and advice in You \& Your Brain, a web series hosted by Prevention, HealthyWomen, and the Women's Alzheimer's Movement.

So Brinton and a number of her colleagues around the world started focusing on what might be going on specifically in women's brainsbeyond aging and unlucky genes - to lead to such higher rates of brain disease.

\section{Human rights}

Unfortunately, people with dementia are frequently denied the basic rights and freedoms available to others. In many countries, physical and chemical restraints are used extensively in care homes for older people and in acute-care settings, even when regulations are in place to uphold the rights of people to freedom and choice.

An appropriate and supportive legislative environment based on internationally-accepted human rights standards is required to ensure the highest quality of care for people with dementia and their carers.

\section{WHO recognizes dementia as a public health priority}

WHO recognizes dementia as a public health priority. In May 2017, the World Health Assembly endorsed the Global action plan on the public health response to dementia 2017-2025. The Plan provides a comprehensive blueprint for action - for policy-makers, international, regional and national partners, and WHO as in the following areas: addressing dementia as a public health priority; increasing awareness of dementia and creating a dementia-inclusive society; reducing the risk of dementia; diagnosis, treatment and care; information systems for dementia; support for dementia carers; and, research and innovation

An international surveillance platform, the Global Dementia Observatory (GDO), has been established for policy-makers and researchers to facilitate monitoring and sharing of information on dementia policies, service delivery, epidemiology and research. As a complement to the GDO, WHO launched the GDO Knowledge Exchange Platform, which is a repository of "good practices" in the area of dementia with the goal of fostering multi-directional exchange between regions, countries and individuals to facilitate action globally.

WHO has developed Towards a dementia plan: a WHO guide plan: a WHO guide, which provides guidance to Member States in creating and operationalizing a dementia plan. The guide is closely linked to WHO's GDO and includes associated tools such as a checklist to guide the preparation, development and implementation of a dementia plan. It can also be used for stakeholder mapping and priority setting.

WHO's Guidelines on risk reduction of cognitive decline and dementia provide evidence- based recommendations on interventions for reducing modifiable risk factors for dementia, such as physical inactivity and unhealthy diets, as well as controlling medical conditions linked to dementia, including hypertension and diabetes. The recently released mDementia handbook provides guidance on implementing mHealth programmes such as two-way messaging using mobile phone technology, which also contains a module and message libraries on dementia risk reduction.

Dementia is also one of the priority conditions in the WHO Mental Health Gap Action Programme (mhGAP), which is a resource for generalists, particularly in low- and middle-income countries, to help them provide first-line care for mental, neurological and substance use disorders.

WHO's iSupport, a knowledge and skills training programme for carers of people living with dementia is available as an online course and a hardcopy manual. iSupport Lite includes easy-to-read posters and a brief video that can act as a quick reference or a refresher, reinforcing previously-acquired caregiving skills and knowledge.

In July 2021, WHO released "Towards a dementia-inclusive society: WHO toolkit for dementia-friendly initiatives", which to support countries in establishing, scaling and evaluating dementia-friendly initiatives to foster societies where people with dementia and their carers can meaningfully participate.

WHO is also developing a Dementia Research Blueprint, together with researchers and academics around the world, to synergize efforts and harmonize the global dementia research and innovation agenda.

\section{Conclusions}

Only a quarter of countries worldwide have a national policy, strategy or plan for supporting people with dementia and their families, according to the WHO's 'Global status report on the public health response to dementia', released today. Half of these countries are in WHO's European Region, with the remainder split between the other Regions. Yet even in Europe, many plans are expiring or have already expired, indicating a need for renewed commitment from governments.

At the same time, the number of people living with dementia is growing according to the report: WHO estimates that more than 55 million people ( $8.1 \%$ of women and $5.4 \%$ of men over 65 years) are living with dementia. This number is estimated to rise to 78 million by 2030 and to 139 million by 2050 .

Dementia is caused by a variety of diseases and injuries that affect the brain, such as Alzheimer's disease or stroke. It affects memory and other cognitive functions, as well as the ability to perform everyday tasks. The disability associated with dementia is a key driver of costs related to the condition. In 2019, the global cost of dementia was estimated to be US\$ 1.3 trillion. The cost is projected to increase to US\$ 1.7 trillion by 2030 , or US\$2.8 trillion if corrected for increases in care costs.

"Dementia robs millions of people of their memories, independence and dignity, but it also robs the rest of us of the people we know and love," said Dr Tedros Adhanom Ghebreyesus, Director-General of the World Health Organization. "The world is failing people with dementia, and that hurts all of us. Four years ago, governments agreed a clear set of targets to improve dementia care. But targets alone are not enough. We need concerted action to ensure that all people with dementia are able to live with the support and dignity they deserve."

\section{More support needed, particularly in low- and middle- income countries}

The report highlights the urgent need to strengthen support at national level, both in terms of care for people with dementia, and in support for the people who provide that care, in both formal and informal settings.

Care required for people with dementia includes primary health care, specialist care, community-based services, rehabilitation, long-term care, and palliative care. While most countries (89\%) reporting to WHO's Global Dementia Observatory say they provide some community-based services for dementia, provision is higher in high-income countries than in low- and middle-income countries. Medication for dementia, hygiene products, assistive technologies and household adjustments are also more accessible in high-income countries, with a greater level of reimbursement, than in lower-income countries.

The type and level of services provided by the health and social care sectors also determines the level of informal care, which is primarily provided by family members. Informal care accounts for about half the global cost of dementia, while social care costs make up over a third. In low- and middle-income countries, most dementia care costs are 
attributable to informal care (65\%). In richer countries informal and social care costs each amount to approximately $40 \%$.

In 2019, carers spent on average five hours a day providing support for daily living to the person they were caring for with dementia; $70 \%$ of that care was provided by women. Given the financial, social and psychological stress faced by carers, access to information, training and services, as well as social and financial support, is particularly important. Currently, $75 \%$ of countries report that they offer some level of support for carers, although again, these are primarily high-income countries.

\section{New initiative to better coordinate dementia research}

A series of unsuccessful clinical trials for treatments for dementia, combined with the high costs of research and development, led to declining interest in new efforts. There has, however, been a recent increase in dementia research funding, mainly in high-income countries such as Canada, the United Kingdom and the United States of America. The latter increased its annual investment in Alzheimer's disease research from US\$ 631 million in 2015 to an estimated US\$ 2.8 billion in 2020.

"To have a better chance of success, dementia research efforts need to have a clear direction and be better coordinated," said Dr Tarun Dua, Head of the Brain Health Unit at WHO. "This is why WHO is developing the Dementia Research Blueprint, a global coordination mechanism to provide structure to research efforts and stimulate new initiatives." An important focus of future research efforts should be the inclusion of people with dementia and their carers and families. Currently two-thirds of countries reporting to the Global Dementia Observatory involve people with dementia "rarely" or not at all.

\section{Good progress in awareness-raising campaigns}

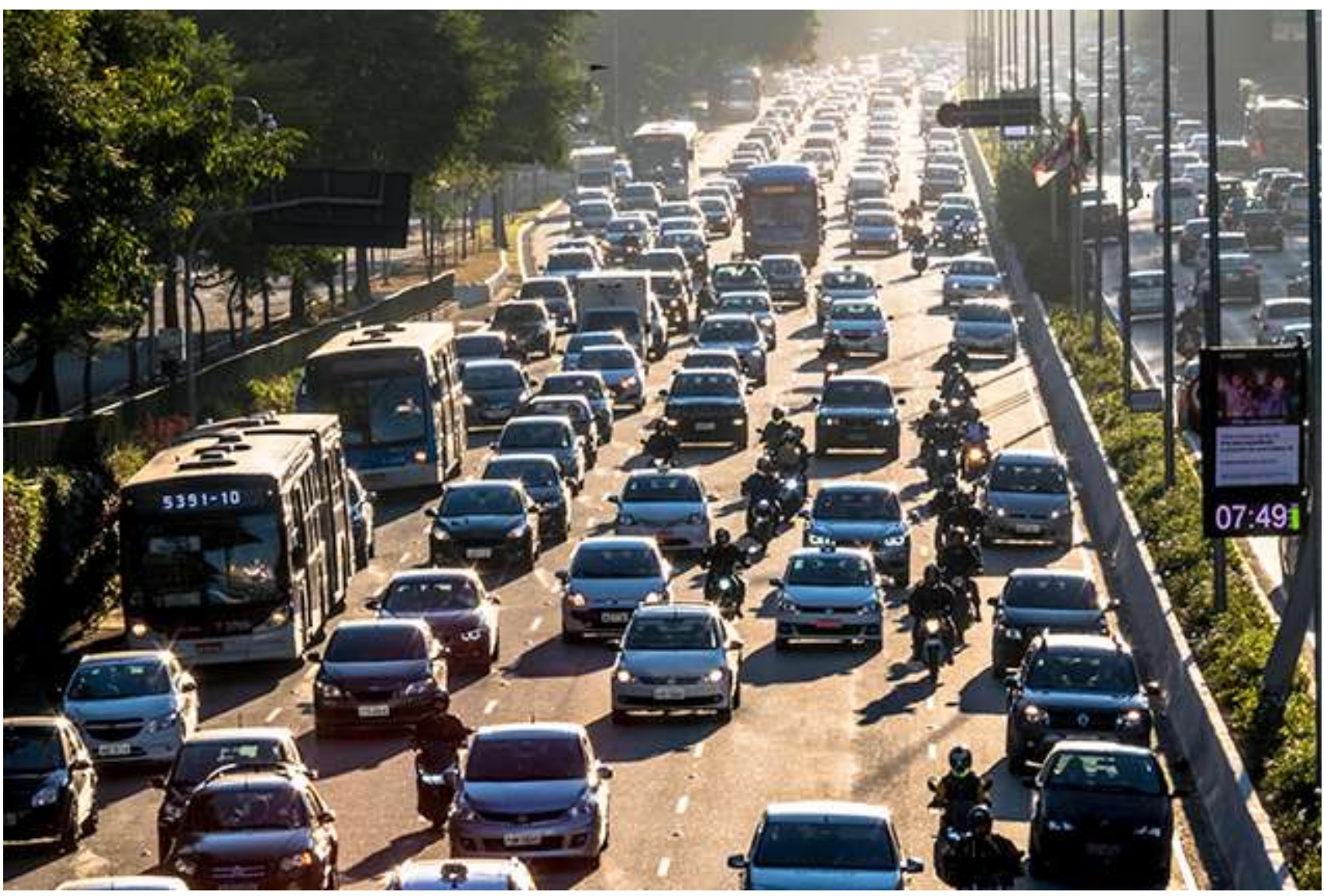

Urban areas are centers of bustling activity, which can provide ample opportunities for cognitively stimulating activities, but also increase
More positively, countries in all regions have made good progress in implementing public awareness campaigns to improve public understanding of dementia, with strong leadership by civil society. Twothirds of countries reporting to the Observatory have run awarenessraising campaigns. And two-thirds have taken action to improve the accessibility of physical and social environments for people with dementia and to provide training and education to population groups outside the health and social care sector, such as volunteers, police, fire services and first responders.

\section{Public health response to dementia}

The 'Global status report on the public health response to dementia' takes stock of progress made to date towards the 2025 global targets for dementia laid out in the WHO's 'Global Dementia Action Plan' published in 2017. It uses data from WHO's Global Health Estimates 2019 and the Global Burden of Disease study 2019 as well as from WHO's Global Dementia Observatory (GDO). So far, 62 countries have submitted data to the GDO, $56 \%$ of which are high-income and $44 \%$ low- and middleincome. Together, these countries represent $76 \%$ of people aged 60 years or older. Data are included on issues ranging from national policies and diagnosis, treatment and care, to support for carers and research and innovation.

WHO's work on the 'Global dementia action plan', including the Global Dementia Observatory, has been supported by the Consumers, Health, Agriculture and Food Executive Agency (CHAFEA, an Executive Agency of the European Union), the Governments of Germany, Japan, the Netherlands, Switzerland and the United Kingdom, and the Public Health Agency of Canada.

\section{Loud Noises harm the brain}

exposure to excessive noise, commonly referred to as noise pollution. Exposure to noise can lead to short term impairments in cognitive 
function, particularly with respect to the ability to focus and remember, while some studies suggest that, similar to air pollution, chronic exposure to noise pollution may increase the risk for dementia.

A report by the European Environmental Agency found that 12,000 premature deaths and 48,000 cases of ischemic heart disease were attributable to environmental noise pollution each year in Europe [1]. The majority of the noise pollution is related to traffic. Sound is measured on a decibel $(\mathrm{dB})$ scale where zero decibels is the softest sound a human can detect and the intensity of the sound doubles every three decibels [2]. For health measures, sound levels are often reported as $\mathrm{dB}(\mathrm{A})$, which is adjusted to account for how well humans can hear different sound wave frequencies. Advisory guidelines use the threshold of 50 to $55 \mathrm{~dB}(\mathrm{~A})$ to classify environmental noise levels that are potentially hazardous to human health [3]. According to the Environmental Protection Agency, noise levels above $70 \mathrm{~dB}(\mathrm{~A})$ can lead to hearing loss [2].

In a study of 5,227 residents of the Chicago area, global cognitive performance varied with the local neighborhood noise level [4]. The residential noise levels varied from 51.1 to $78.2 \mathrm{~dB}(\mathrm{~A})$. Each $10 \mathrm{~dB}(\mathrm{~A})$ increase in residential noise level was associated with a $36 \%$ increase in the risk for mild cognitive impairment (MCI) and a $29 \%$ increase in the risk for Alzheimer's disease. A study of 4,086 residents in Germany found a similar association regarding the increased risk for MCI in participants exposed to the highest residential noise levels, or greater than $60 \mathrm{~dB}(\mathrm{~A})$ [5]. Based on the assessment of 130,978 residents of London, those exposed to the highest noise levels, greater than $53.8 \mathrm{~dB}$, during the night had a $9 \%$ higher risk of developing dementia [6].

In the majority of cases, residents living in areas with the highest levels of noise pollution were also exposed to higher levels of air pollution and were more likely to have lower socioeconomic status. Since all of these factors impact dementia risk, it can be difficult to tease out the exact contribution of noise. The evidence suggests that each of these factors acts as a stressor, and that it is the lifetime burden of all stressors that influences overall dementia risk [7].

However, noise is a rather unique stressor in that the response to a particular sound stimulus is very subjective because it depends on whether a person perceives the sound as bothersome [3]. If someone is bothered by a sound, then it can induce a stress response in the body and lead to negative health effects. But, if someone can easily tune out the sound, then it is unlikely to produce ill effects in that person. The response to a sound depends on the type, duration, and loudness of the sound itself, as well as factors intrinsic to the person, such as personality. People high in the personality trait of neuroticism, who are more prone to anxiety and depression, tend to be more sensitive to noise and are more likely to experience negative effects following noise exposure [3].

Noise acts as a stressor by inducing a state of arousal in the body, which increases levels of stress hormones, such as cortisol. The brain is wasting resources on trying to tune out the noise, so the brain has less capacity to perform other complex tasks, leading to a temporary decline in cognitive performance [3]. Attention and memory tend to be the cognitive domains most impacted by noise. The stress response leads to vascular changes that can pave the way for cardiovascular disease and vascular dementia. Meta-analyses indicate that each $10 \mathrm{~dB}(\mathrm{~A})$ increase in environmental noise increases the risk for adverse cardiovascular outcomes, including hypertension and heart attacks, by 7 to $17 \%[\mathbf{8}]$. Due to the strong connection between heart health and brain health, these negative effects on the cardiovascular system may account for some of the increased dementia risk from noise pollution. Exposure to noise during the night can impair sleep quality, which has additional negative health consequences. Chronic activation of the arousal-stress response can also cause oxidative stress, which is another driver of dementia.

Noise may be particularly problematic for people with dementia because they do not have enough brain resources to filter the noise and perform other cognitive functions at the same time. Individuals with dementia may need a period of uninterrupted silence to allow them to fully utilize their limited cognitive resources, which may lead to more meaningful interactions.

Since moving away from an area of high noise pollution is not a feasible solution for most people, the best solution for reducing the health hazards of noise involves practicing stress mitigation techniques, such as meditation or yoga.

\section{References}

1. European Environmental Agency (2020) Environmental Noise in Europe.

2. Centers for Disease Control and Prevention (2019) Loud Noise Can Cause Hearing Loss.

3. Wright B, Peters E, Ettinger U et al. (2014) Understanding noise stress-induced cognitive impairment in healthy adults and its implications for schizophrenia. Noise and Health 16, 166-176.

4. Weuve J, D'Souza J, Beck T et al. (2020) Long-term community noise exposure in relation to dementia, cognition, and cognitive decline in older adults. Alzheimer's \& Dementia.

5. Tzivian L, Dlugaj M, Winkler A et al. (2016) Long-Term Air Pollution and Traffic Noise Exposures and Mild Cognitive Impairment in Older Adults: A Cross-Sectional Analysis of the Heinz Nixdorf Recall Study. Environmental Health Perspectives 124, 1361-1368.

6. Carey IM, Anderson HR, Atkinson RW et al. (2018) Are noise and air pollution related to the incidence of dementia? A cohort study in London, England. BMJ Open 8, e022404.

7. Paul KC, Haan M, Mayeda ER et al. (2019) Ambient Air Pollution, Noise, and Late-Life Cognitive Decline and Dementia Risk. Annual Review of Public Health 40, 203220.

8. Basner M, Babisch W, Davis A et al. (2014) Auditory and non auditory effects of noise on health. The Lancet 383, 1325-1332.

9. More about dementia

10. The Global Dementia Observatory

11. iSupport

12. WHO mhGAP Intervention Guide 